\title{
Raman spectra and structural phase transition in $\operatorname{Pr}_{3} \mathrm{Sb}_{5} \mathrm{O}_{12}$ crystal
}

Abdumalik Hojiboev ${ }^{1}$, Aleksandr Oreshonkov ${ }^{*, 2,3}$, Maksud Umarov ${ }^{4}$, Alexander Vtyurin 2,3

${ }^{1}$ Department of Theoretical Physics, Khujand State University, 735700, Khujand, Tajikistan

${ }^{2}$ Laboratory of Molecular Spectroscopy, Kirensky Institute of Physics, SB RAS, 660036, Krasnoyarsk, Russia

${ }^{3}$ Department of Photonics and Laser Technology, Siberian Federal University, 660079, Krasnoyarsk, Russia

${ }^{4}$ Department of Biomedical Engineering, Vologda State University, 160000, Vologda, Russia

*Corresponding author: oreshonkov@iph.krasn.ru, Phone: +7 391249 4510, Fax: +7 3912438923 


\title{
Raman spectra and structural phase transition in $\operatorname{Pr}_{3} \mathrm{Sb}_{5} \mathrm{O}_{12}$ crystal
}

\author{
Raman spectroscopy investigation of phase transition in $\mathrm{Pr}_{3} \mathrm{Sb}_{5} \mathrm{O}_{12}$ crystal \\ is reported. Spectra were obtained in temperature range from 300 to 800 \\ K. Soft mode restoration has been found below $735 \mathrm{~K}$, that allows to \\ attribute structural phase transition as displacive.
}

Keywords: antimonite; praseodymium; soft mode; raman spectroscopy

\section{Introduction}

Oxides of trivalent antimony belongs to the family of antimonite crystals $R_{3} \mathrm{Sb}_{5} \mathrm{O}_{12}$ where $R$ is rare-earth ions [1-3]. This family of crystals has received special attention because of their ferroelectric and piezoelectric properties $[4,5]$. The potential luminescence properties have been studied in gadolinium antimonite [6]. Unit cell structure of $R_{3} \mathrm{Sb}_{5} \mathrm{O}_{12}$ crystal family at room temperature is shown in Fig. 1 (space group $I-43 m, Z=4$ [2]). As can be seen from Fig. 1, rare-earth cations are surrounded by 8 oxygen anions, i. e. situated in polyhedra with eight vertices and can be presented as "Thomson folded cube". Three-dimensional crystal framework is formed by polyhedrons linked to each other by common edges. Sb atoms are located in cavities of the crystal structure.

Crystals of antimonite family undergoes a structural phase transition to high temperature non-centrosymmetric cubic phase Im3m; such transitions for $\mathrm{Nd}_{3} \mathrm{Sb}_{5} \mathrm{O}_{12}$, $\mathrm{Gd}_{3} \mathrm{Sb}_{5} \mathrm{O}_{12}$ and $\mathrm{Er}_{3} \mathrm{Sb}_{5} \mathrm{O}_{12}$ crystals were found at 723,713 and $643 \mathrm{~K}$, correspondently [7]. The temperature dependence of dielectric constant of $R_{3} \mathrm{Sb}_{5} \mathrm{O}_{12}(R=\mathrm{Pr}, \mathrm{Nd}, \mathrm{Gd}, \mathrm{Er})$ have been studied in [8]; it has been shown that $\varepsilon(\mathrm{T})$ of $\mathrm{Pr}_{3} \mathrm{Sb}_{5} \mathrm{O}_{12}$ has a maximum at $740 \mathrm{~K}$ as well, that can indicate the presence of structural phase transition. Possible existence of the structural phase transition in this temperate region was discussed earlier 
in [9]. Raman spectra of $\operatorname{Pr}_{3} \mathrm{Sb}_{5} \mathrm{O}_{12}$ crystal at room temperature have been obtained in [10], but its temperature dependence has not been studied yet.

The aim of this work was to perform the investigation of Raman spectra of $\operatorname{Pr}_{3} \mathrm{Sb}_{5} \mathrm{O}_{12}$ crystal in a wide temperature range looking for some features of such structural phase transition.

\section{Experimental technique and results}

Raman spectra were collected using the polarized radiation of a $632.8 \mathrm{~nm}$ Helium-Neon (He-Ne) laser $(50 \mathrm{~mW})$. The spectra in the $90^{\circ}$ geometry were recorded with DFS-24 Raman spectrometer in the wavenumber range $20-1200 \mathrm{~cm}^{-1}$ and instrument function width $0.5 \mathrm{~cm}^{-1}$. The temperature studies were performed using a high temperature thermostat [11] in 300-800 K range; the accuracy of temperature stabilization during spectra measurement was $<0.1 \mathrm{~K}$.

Samples for experiments $3 \times 4 \times 6 \mathrm{~mm}^{3}$ were optically transparent and without colored defects or inclusions visible under the microscope. High quality of samples was confirmed by acoustic quality factor measurements.

Vibration representation of the $I-43 m$ cubic phase at Brillouin zone center is: $\Gamma_{\text {vibr }}(I-43 m)=6 A_{1}(x x, y y, z z)+3 A_{2}+9 E(x x, y y, z z)+13 F_{1}+18 F_{2}(x y, x z, y z)$, where corresponding components of the Raman scattering tensor are given in brackets. The polar $F_{2}$ modes could be split into the LO and TO components [9]. Vibration representation of the expected high temperature phase at Brillouin zone center is: $\Gamma_{\text {vibr }}(\operatorname{Im} 3 m)=3 A_{1 \mathrm{~g}}(x x, y y, z z)+2 A_{2 \mathrm{~g}}+5 E_{g}(x x, y y, z z)+6 F_{1 \mathrm{~g}}+7 F_{2 \mathrm{~g}}(x y, x z, y z)+A_{1 \mathrm{u}}+$ $3 A_{2 \mathrm{u}}+4 E_{\mathrm{u}}+7 F_{2 \mathrm{u}}+11 F_{1 \mathrm{u}}$ 
Raman spectra of $\operatorname{Pr}_{3} \mathrm{Sb}_{5} \mathrm{O}_{12}$ crystal $(T=300 \mathrm{~K})$ at $x(z z) y$ and $z(y x+y z) z$ geometries are shown in Fig. 2; $A_{1}$ и $E$ modes can be detected in $x(z z) y$ geometry, $F_{2}$ modes can be observed in $z(y x+y z) z$ geometry only.

The number of observed Raman modes is less then allowed by the selection rules, probably due to sufficiently large widths of the bands at room temperature and their strong overlapping.

Temperature transformation of Raman spectra in $x(z z) y$ geometry is shown in Fig. 3. Crystal heating leads to further increasing of line widths, and to disappearance of some Raman lines in accordance with the selection rules above the phase transition temperature.

Important spectral feature is found in the $x(z z) y$ diagonal component. The frequency of Raman band at $114 \mathrm{~cm}^{-1}$ (at $T=300 \mathrm{~K}$ ) rapidly shifts toward the Rayleigh wing. The temperature dependence of squared mode wavenumber (Fig. 4) is typical for soft modes behavior. Extrapolation of this dependence to zero gives the value of the critical temperature at $735 \mathrm{~K}$ that agrees with observed temperature of maximum of dielectric constant $(740 \mathrm{~K})$ and corresponds to the second order phase transition. Group-theory analysis of the eigenvectors of the normal vibrations in $I-43 m$ phase shows that $\mathrm{O}$ and $\mathrm{Sb}$ ions are involved in the eigenvectors of $A_{1}$ lattice vibrations, i. e. this phase transition is associated with the displacement of these ions. This fact can explain weak dependence of the transition temperature on the rare-earth ion.

There is no mode condensation in Raman spectra above the transition point. Grouptheory analysis shows that phase transition $\operatorname{Im} 3 m-I-43 m$ connected with the $\tau 4(\mathrm{k} 11)-$ $A_{2 \mathrm{u}}$ irreducible representation. Modes of such symmetry are inactive in optical vibrational spectra.

It should be noted that the large width of the Raman lines presents in all studied 
temperature range, not only near phase transition point. This fact may indicate large anharmonicity of lattice vibrations of the crystal and large amplitudes of atomic vibrations. Perhaps, crystal structure is partially disordered below the phase transition point, at another phase transition is possible at lower temperatures.

\section{Conclusion}

As a result of these investigations, we can conclude that $\mathrm{Pr}_{3} \mathrm{Sb}_{5} \mathrm{O}_{12}$ crystal undergoes the structural phase transition at $T=735 \mathrm{~K}$, and this transition is accompanied by soft mode restoration. This fact allows us to attribute it to the phase transitions of displacive type. Phase transition is associated with the displacement of $\mathrm{O}$ and $\mathrm{Sb}$ ions.

\section{Acknowledgements}

A.S.O. and A.N.V. are partially sup-ported by the Ministry of Education and Science of the Russian Federation.

\section{References}

[1] Kh.M. Kurbanov, M.N. Tseitlyn, R.Ch. Bychurin and etc., Dokl. Akad. Nauk RT, 24, 494 (1981). (in Russian)

[2] Kh.M. Kurbanov, N.B. Butikova, A.G. Gukalova, V.P. Glyakin, Dokl. Akad. Nauk SSSR, 281, 1119 (1985). (in Russian)

[3] A.G. Gukalova, V.P. Glyakin, M.N. Tseitlyn, Koordinacion-naya himiya, 13, 918 (1987). (in Russian)

[4] M.F. Umarov, A.N. Vtyurin, A.K. Hojibaev, Proceedings Order disorder and properties of oxydes ODPO-13,(Rostov-on-Don, 2010), pp. 178. (in Russian)

[5] M.F. Umarov, K.S. Koziev, A.K. Hojibaev, Uchenie zapiski HGU, 2, 58 (2012). (in Russian)

[6] G. Blasse and H.S. Kiliaan. Inorg. chim. Acta 117. L23 I 
[7] Kh.M. Kurbanov, M.N. Tseitlyn,, I.P. Raevskii, Izv. Akad. Nauk SSSR

Neorganicheskie materiali, 20, 1199 (1984). (in Russian)

[8] M.F. Umarov, A.K. Hojibaev, K.S. Koziev, S.Sh. Ahmedov, Vestn. Taj. Nac. Univ., 1/2(106), 150 (2013). (in Russian)

[9] V.P. Glyakin, Pn.D. dissertation, Dushanbe, Akad. Nauk Taj. SSR, phys. Tech. Univ., 1989.

[10] I.L. Botto, E.J. Baran, C. Cascales, I. Rasines, R. Saez Puche, Journal of Physics and Chemistry of Solids, 52, 431 (1991).

[11] Z.M Rahmatova, M.F. Umarov, A.K. Hojibaev, Vestn. Taj. Tech.Univ, 2(22), 4 (2013). (in Russian) 
Fig. 1: Structure of $\mathrm{Pr}_{3} \mathrm{Sb}_{5} \mathrm{O}_{12}$ crystal at room temperature

Fig. 2: Raman spectra of $\operatorname{Pr}_{3} \mathrm{Sb}_{5} \mathrm{O}_{12}$ at $x(z z) y$ and $z(y x+y z) y$ geometry

Fig. 3: Temperature transformation of Raman spectra of $\mathrm{Pr}_{3} \mathrm{Sb}_{5} \mathrm{O}_{12}$ crystal

Fig. 4. Temperature dependence of squared soft mode wavenumber. 


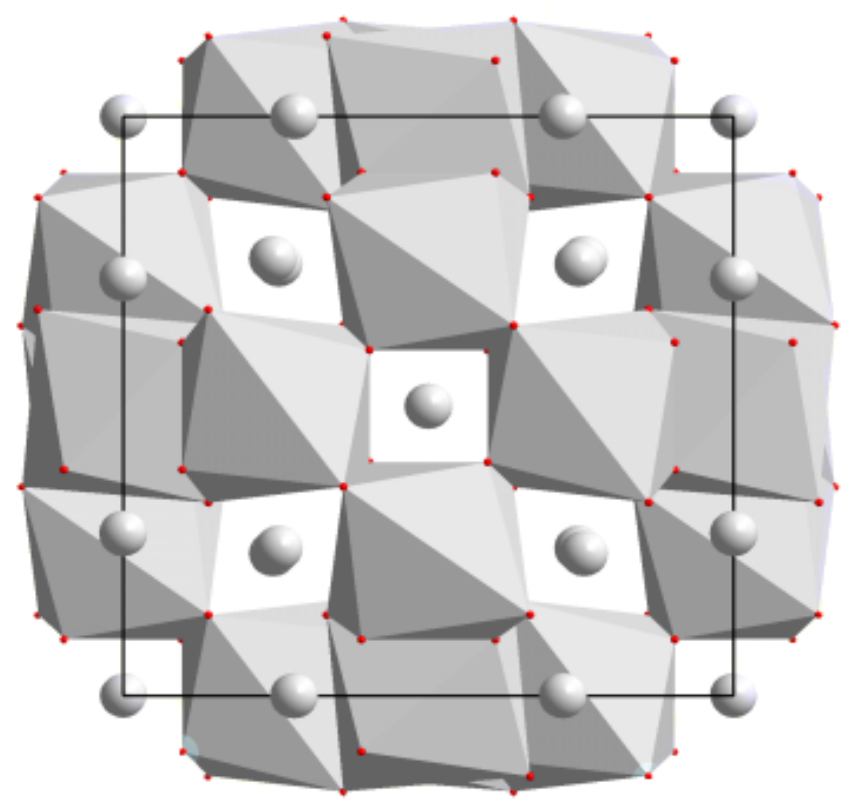

Fig.1. 


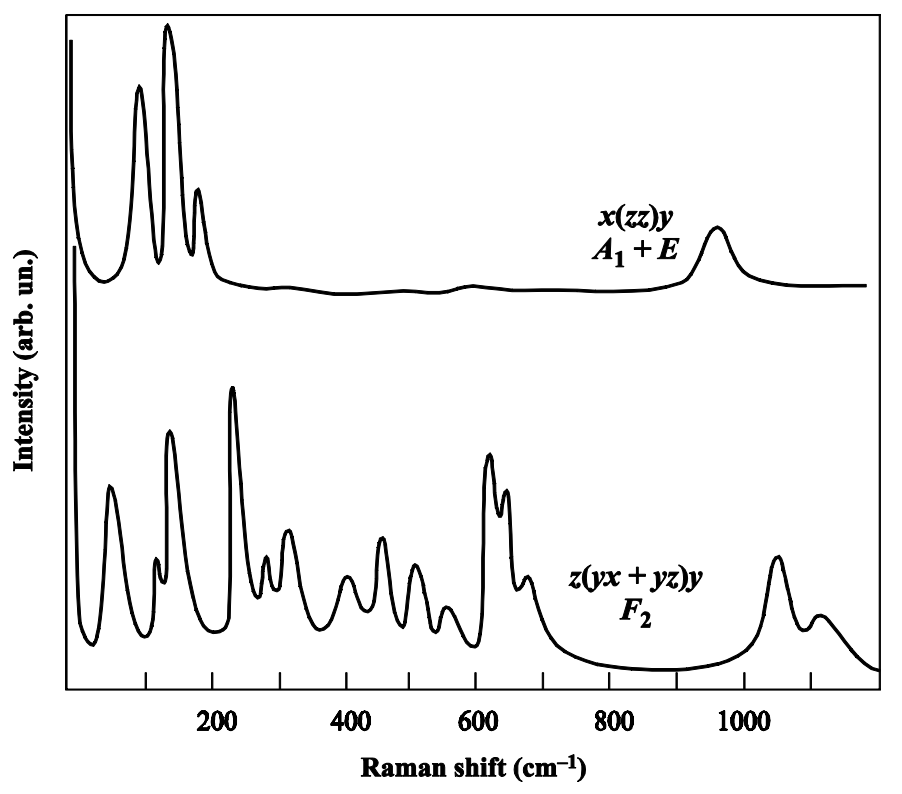

Fig.2. 


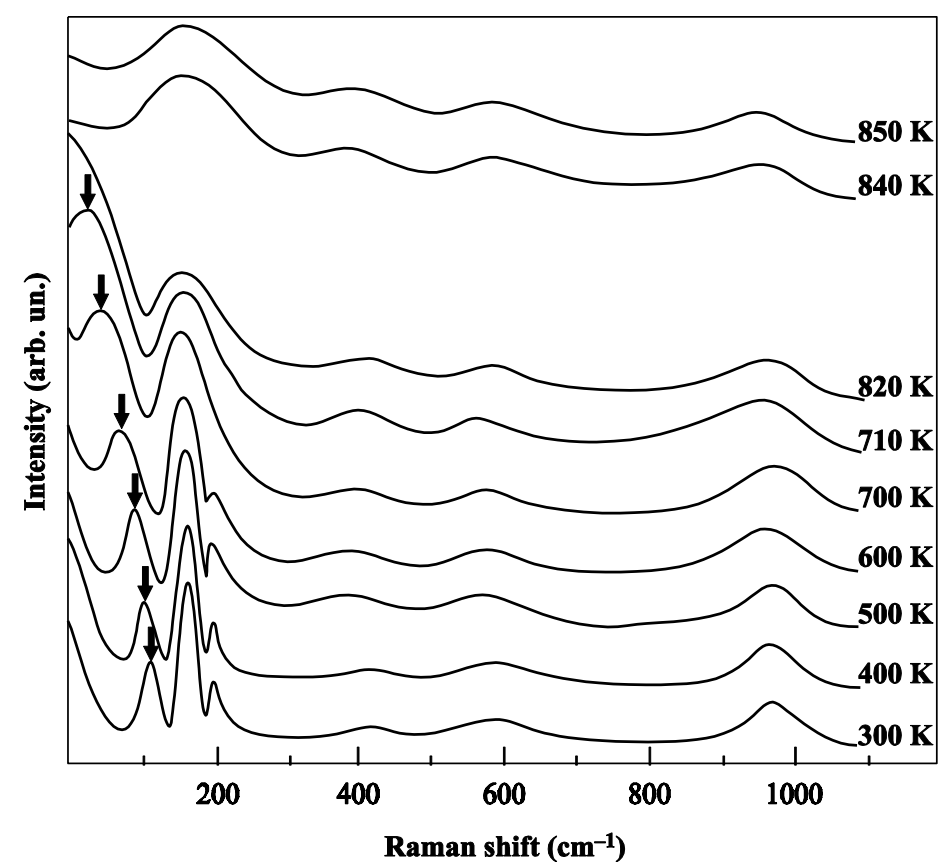

Fig. 3. 


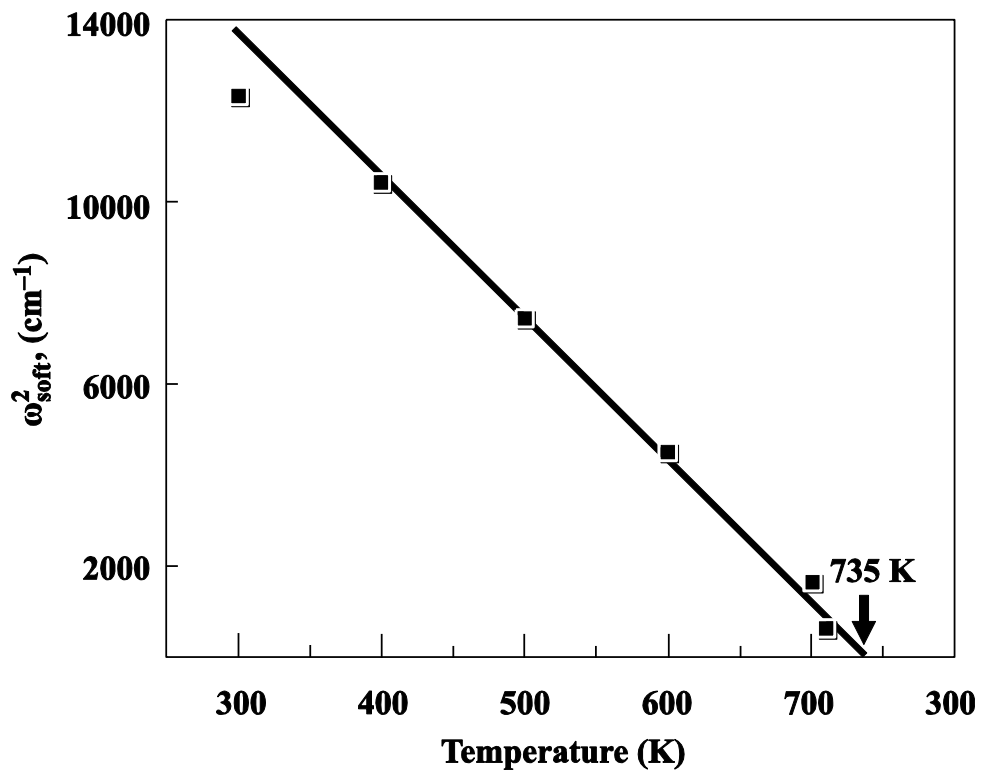

Fig.4. 